The Be Phenomenon in Early-Type Stars, IAU Colloquium 175

ASP Conference Series, Vol. 214, 2000

M. A. Smith, H. F. Henrichs, and J. Fabregat, eds.

\title{
Line Profile Modeling of Disks
}

\author{
W. Hummel \\ Institut für Astronomie und Astrophysik und Universitätssternwarte \\ München, Scheinerstr 1, D-81673 München, Germany
}

\begin{abstract}
In this talk I give a short overview of models for spectral lines in Be stars disks which I think are quite instructive. I then examine the arguments for my own model assumptions and discuss selected topics concerning radiative line transfer calculations in three dimensions. We show that symmetric $\mathrm{H} \alpha$ emission lines of Be stars can be understood in terms of Keplerian disks. It is also demonstrated that one-armed global disk oscillations provide the best available solution to understand the long-term $V / R$ variations in Be star emission lines.
\end{abstract}

\section{Introduction}

It is the dream of every astronomer to derive astrophysical properties directly from the observations. In all cases where this dream has come true the underlying interface between, e.g. the spectroscopic classification and the physical parameters are models with certain predictions e.g. of line ratios.

For the Be star disks the most striking feature in the optical are the Balmer emission lines. Though emission lines of Be stars show a wide range of profile shapes they can nevertheless be classified by simple criteria (Hanuschik et al. 1996 and references therein). About 3/4 of all galactic field Be stars show symmetric line profiles with strengths and profile shapes almost constant over years (= class 1$)$. The profiles of the remaining stars exhibit asymmetric emission lines variable in a cyclic manner on the timescale of a decade (=class 2). Representative examples are given in Fig. 1.

An early interpretation of these profiles was given by Struve (1931) who argued that:

... rapidly rotating single stars of spectral class $B$ are unstable and form lens-shaped bodies which eject matter at the equator, thus forming a nebulous ring which revolves around the star and gives rise to the emission lines...

The main argument for this interpretation is the observed correlation between the projected stellar rotation $v \sin i$ (a stellar parameter from atmospheric absorption profiles) and the FWHM of the emission lines which is interpreted as due to kinematical broadening. Assuming that all Be stars have nearly the same rotational velocity, the scatter of $v \sin i$ from star to star is mainly caused by different aspects, $\sin i$. Striking "flank inflections" (Fig.1.) are most pronounced in large equivalent width $\mathrm{H} \alpha$ profiles of Be stars with low inclination. This feature is less developed in higher members of the Balmer series and is completely absent in emission lines of $\mathrm{Fe} I \mathrm{I}$ and $\mathrm{He}$. 


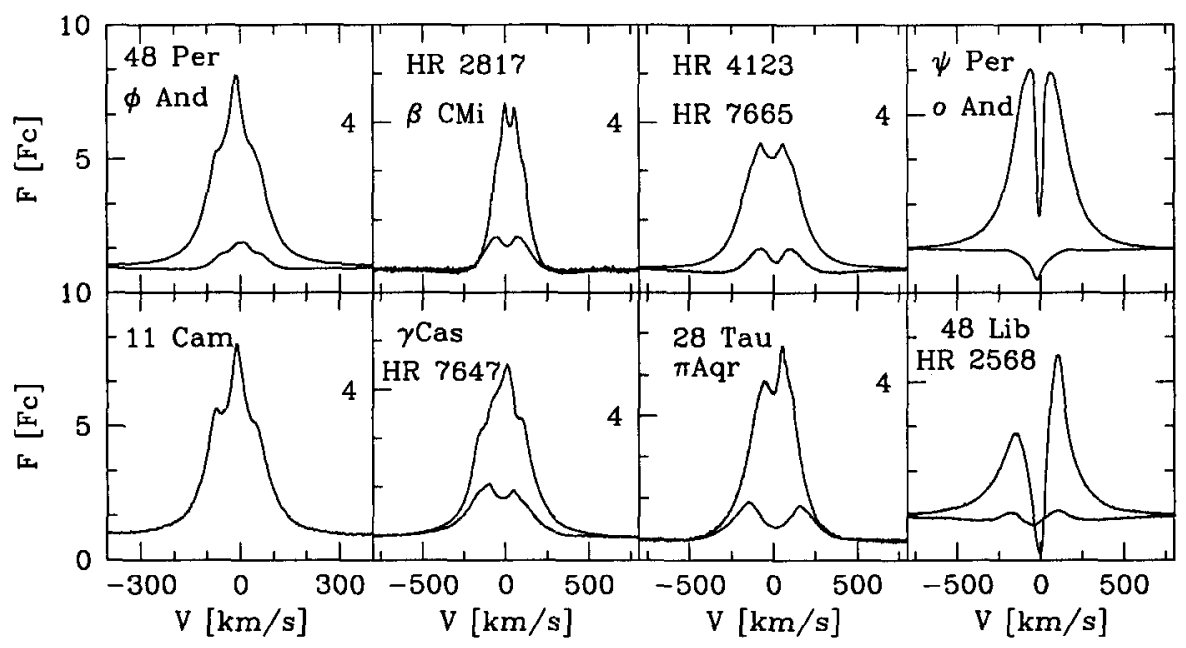

Figure 1. Representative high resolution line profiles of Be stars. Upper row: class 1 profiles, symmetric and rather constant on a timescale of years. The stellar $v \sin i$ is increasing from left to right. Lower row: class 2 profiles, asymmetric and cyclical variable on a time scale of a decade. Bumps in the wings (=flank inflections) are most pronounced in high equivalent width profiles at low $v \sin i$.

Apart from these two main types of emission line appearance, there are some variability patterns which do not fit into neat classes. Most of these are associated with either Be binary stars (e.g. $\phi$ Per, HR 2142), Be X-ray binaries, or the formation and destruction of circumstellar disks (e.g $\mu$ Cen, $\lambda$ Eri). In this study we will focus on the structure and evolution of stationary circumstellar disks of single Be stars.

\section{A short history}

Creating useful model requires imagination, both to construct the model itself and to impose realistic simplifications. The simplest class of models for emission lines of Be stars are those of Smak (1969), Huang (1972) and Hanuschik (1988). The main assumptions are optically thin line radiation and kinematical broadening in a differentially rotating disk. These models are sufficient to explain the double peak structure of intermediate inclination profiles of Fe II. However, they fail to reproduce the complex structure of $\mathrm{H} \alpha$ and shell profiles. Horne \& Marsh (1986) introduced a straightforward method to include opacity effects in a Keplerian disk:

Line photons tend to emerge from an optically thick emission layer in directions along which the Keplerian velocity field of the disk provides a velocity gradient. 
This velocity shear can explain the $V$-shape of symmetric line profiles due to self absorption in a Keplerian disk. To model shell profiles the finite size of the central star must be taken into account (Hummel \& Vrancken, these proc.).

Among the disk models which include multi-level model atoms (for a review see Poeckert, 1982), the Poeckert \& Marlborough (1978) model is the most elaborate. Although the assumed velocity field, in particular the outflow velocity in the disk, is currently questionable, the Poeckert \& Marlborough model agrees well with the observed degree of polarization, with the Balmer decrement, and with the formation of the line profile wings due to electron scattering. Recent improvements and applications of this model are given by Waters (these proc.) and Millar \& Marlborough (these proc.).

\section{Observational constrains and model assumptions}

Since our model assumptions, in particular the velocity field, is at variance to the assumption of an outflowing disk, we will briefly collect the relevant observational constraints (e.g. Telting, these proc.) for those assumptions.

\subsection{Density distribution}

Be stars form a group of fast rotators. The distribution of the intrinsic stellar rotation peaks at $70 \%$ of the theoretical break-up velocity. The observed distribution of $v \sin i$ mainly represents the random distribution of the rotation axis. The correlation between the FWHM of line profiles and the $v \sin i$ of a star means that the rotation axis of the circumstellar gas equals that of the central star.

Only Be stars with large $v \sin i$ show many narrow metallic absorption profiles (shell-profiles). This means that the circumstellar gas occults the stellar disk only when seen equator-on. Hence there is a flattened density distribution concentrated towards the equatorial plane of the star. The polarization in emission lines (Poeckert \& Marlborough 1978) as well as interferometric results (Quirrenbach et al. 1997) have verified the disk-like shape distribution of the Be star circumstellar emission region. For the density distribution we use:

$$
N=N_{0} R^{-m} \exp \left(-\frac{z^{2}}{2 H^{2}(R)}\right)
$$

where the radial run of the density is determined by $m$ and the vertical extension of the disk is given by the balance between the thermal gas pressure and the $z$-component of the stellar gravitation. The opening angle in such a disk is not a fixed value but is (for a given value of $m$ ) dependent on the location in the disk (Fig. 2).

\subsection{Kinematics}

The kinematics in the disks has been a matter of debate for a long time. The kinematic interpretation of Doppler shifts in emission and in particular of shell lines are evidence for the absence of expansion velocities larger than the thermal motion in the disk (Hanuschik, these proc.). Since the line-of-sight velocity in 

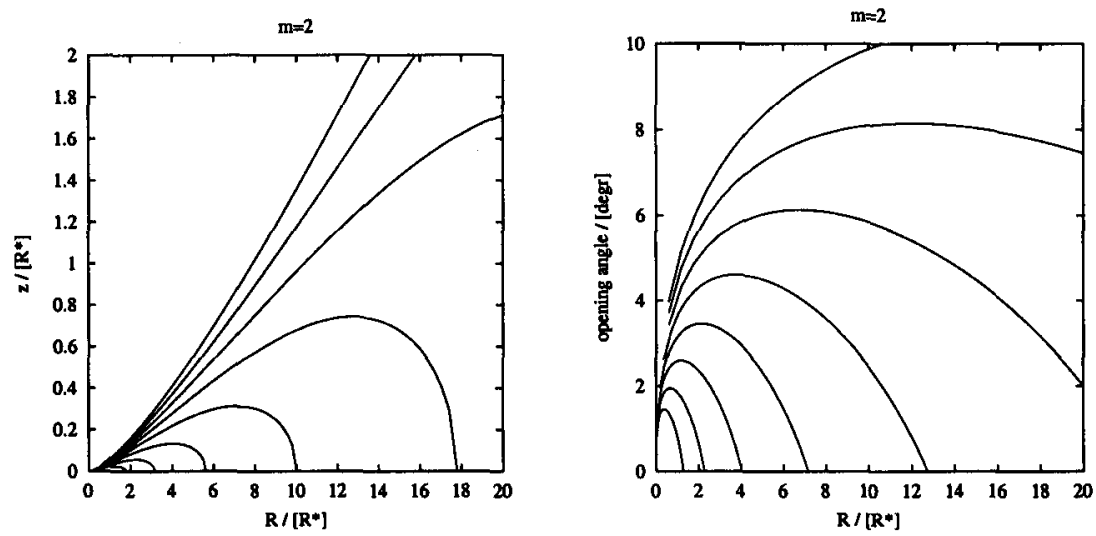

Figure 2. Left: Meridional cross section of the density contours for $m=2$ at $\log \left(N / N_{0}\right)=-0.5,-1,-1.5, \ldots,-4$. Right: opening angle along the density contours for the same $\log \left(N / N_{0}\right)$.

the circumstellar absorption through is zero there is no radial motion in the line formation region. Otherwise we would observed P-Cygni similar profile shapes. The rotation law, parameterized by $R_{\text {rot }}=R^{-j}$, is more difficult to derive from the observations. The comparison between spectroscopic and interferometric $H \alpha$ emission disk radii constrain $j$ to $j<0.65$ (Hummel \& Vrancken, these proc.). This strongly supports Keplerian rotation, Hence we use

$$
V_{\phi}=V_{0} R^{-\frac{1}{2}}, V_{z}=0, V_{R}=0 .
$$

\subsection{Radiation properties}

The spectra of Be stars look like those of ordinary B stars superimposed by prominent emission lines and an IR excess. But edge-on Be-stars (shell stars) like Pleione lack a Balmer continuum and exhibit only about $1 / 3$ of the normal stellar Paschen continuum (Doazan et al. 1987). This means that the stellar UV radiation field is reprocessed in the disk. Hydrogen is ionized and recombines producing the emission lines, while the IR excess represents the thermal free-free and bound-free radiation of the much cooler disk. For our modeling we assume $\mathrm{L} \alpha$ to be optically thick and in detailed equilibrium ("Menzel case B"). Hence $\mathrm{H} \alpha$ behaves approximately like a resonance line and can be modeled by a simple equivalent two-level approach.

\subsection{Numerical aspects of radiative transfer in $3 D$}

Our main intention is to understand the formation of $\mathrm{H} \alpha$ and its relation to the structure and the long-term evolution of Be star disks. The Poeckert \& Marlborough model and the method of Kriz (1978) emphasize complex multilevel model atoms at the cost of geometrical effects. For example, the method of Poeckert \& Marlborough is restricted to axisymmetric disks and the method Kriz does not resolve the vertical structure of the disk. To overcome the geometrical 
restrictions of previous models we have to solve the radiative transfer equation in 3 spatial dimensions. Hence, we take the full geometry into account but at the cost of an elaborate model atom.

The radiative transfer equation in its spatially implicit form is discretized on a three-dimensional Cartesian mesh. In each iteration step for the $\mathrm{H} \alpha$ line source function we determine the specific intensity $I_{x, y, z}(\lambda, \theta, \phi)$ at any gridpoint for about 60 wavelength points around $\mathrm{H} \alpha$ and for all direction angles $(\theta, \phi)$. The mean intensity $\bar{J}$ and the new source function is determined in the framework of an accelerated $\Lambda^{*}$-iteration and using hyperbolic extrapolation methods (Rybicki, 1991 for a review). After convergence of the source function $S$ is obtained, the emergent intensity is determined via the transport equation at the rim of the grid to obtain the H $\alpha$ line profile (see Hummel 1994 and references therein).

\section{Results for axi-symmetric disks}

In a first attempt we calculate $\mathrm{H} \alpha$ model profiles of two Keplerian disks with footpoint densities of $N_{0}=10^{12} \mathrm{~cm}^{-3}$ and $N_{0}=10^{14} \mathrm{~cm}^{-3}$. The radial density parameter is $m=2$ and a $\mathrm{H} \alpha$ emitting disk radius of $R_{\mathrm{d}}=10$ is chosen (Hummel \& Dachs 1992, Hummel 1994). The line profiles of the low density model are dominated by kinematical broadening. At large inclinations $\left(i>60^{\circ}\right)$ selfabsorption in the disk increases the central depression. For $i>70^{\circ}$ the disk obscures a considerable fraction of the central star in a narrow wavelength range producing shell-profiles.

The high density model profiles differ from the low density model profiles by additional bumps in the profile wings sometimes called "flank inflections", which gives the profile the characteristic so-called "winebottle-type" shape, a frequently observed feature (Fig. 1.). This effect can be understood as an additional broadening mechanism, which only becomes apparent when the disk becomes optically thick for line radiation in direction perpendicular to the disk plane. For pole-on view $\left(i=0^{\circ}\right)$ when the kinematical broadening has vanished the remaining low separation double peak profile represents the isolated broadening mechanism, called non-coherent scattering, a long-known result for optically thick slabs:

Line photons are scattered into the line wing so they escape in the deeper layers of the disk where the source function is large. This means that the observer looks deeper into the disk in the line wing where the line source function is larger, thus producing an emission peak off the line center.

Thus, the so-called winebottle-type profiles at $i>0^{\circ}$ are simply the product of this slab effect and the kinematical broadening. Higher members of the Balmer series with less pronounced flank inflections are therefore less optically thick for line radiation in direction perpendicular to the disk plane. Fe II emission lines generally lack flank inflections, hence they are optically thin at low inclinations.

In summary, class $1 \mathrm{H} \alpha$ profiles are dominated by kinematical broadening and broadening due to non-coherent scattering broadening (the slab effect, most pronounced for the pole-on view). No outflow in the disk is required to understand the optical emission lines. Moreover, outflow would result in a general 
blue shift of the shell absorption which is not a common feature of the Be-shell stars.

\section{V/R-variations}

About $1 / 4$ of all galactic field Be stars show asymmetric cyclically variable $\mathrm{H} \alpha$ line profiles (Fig. 1.), the so-called $V / R$-ratio variations, since the intensity ratio of the violet and red emission peak vary. Prominent examples are $\gamma$ Cas, EW Lac, $48 \mathrm{Lib}, \beta^{1}$ Mon. The terminology of class 2 defines the $V / R$ variables as the striking observational appearance of the general long-term cyclic variations at large inclinations.

Both class 2 and class 1 profiles exhibit the basic broadening mechanisms discussed above (e.g. flank inflections, shell profiles), however in a slightly different manner. Moreover some Be stars can change from class 1 to class 2 and vice-versa giving rise to the suggestion that disks of class $2 \mathrm{Be}$ stars are not different physically to those of class 1 Be stars. The mean physical properties (averaged over the entire disk) seem to be retained, since we still observe flank inflections and shell-type profiles in class $2 \mathrm{Be}$ stars, but the detailed distribution inside the disk (e.g density or kinematics) is certainly different with respect to disks showing class $1 \mathrm{H} \alpha$ profiles.

For many years there have been two competing ideas to explain Balmer emission line cyclical variations, the pulsation and the elongated disk models. Predicted line profile variations of the elongated disk model are in better agreement with observations than those of the pulsation model (Ballerau \& Chauville 1989 and references therein). The greatest objection to an elongated disk is the expected circularization of elliptical orbits due to pressure gradients. However, $V / R$ variations are known to continue in the case of $\gamma$ Cas, for example, over more than 30 years. Nevertheless, elongated disk model profiles by Huang (1973) and by Kriz (1978) demonstrate same striking similarities with observations, in particular that the stronger peak has a smaller wavelength offset from line center.

As a new model, Kato (1983) has proposed one-armed density perturbations, a type of a global azimuthal perturbation density pattern which precesses the central star, giving rise to the observed long-term cyclical emission variations. Okazaki (1991) derived eigenfunctions and eigenfrequencies based on linear perturbations in isothermal nearly Keplerian disks influenced by a point mass gravitational potential. Hanuschik et al. (1995) pointed out that class 2 profiles in general agree with the assumption of a large-scale perturbation with . a slowly precessing Keplerian disk.

\subsection{Small disks}

Since retrograde density waves can be trapped within the disk, Okazaki (1991) used an outer disk radius of $5 R_{*}$ as the outer boundary in order to keep the disk oscillation confined. In a first modeling attempt, we have incorporated the eigenfunctions in the density and velocity structures as an underlying disk perturbation and performed 3D radiative line transfer (Hummel \& Hanuschik 1997). The density structure has one strong peak close to the star. After convergence of the line source function (Fig. 3 ) we calculated line profiles but now as a function of inclination and azimuth (Figs. 4,5). 


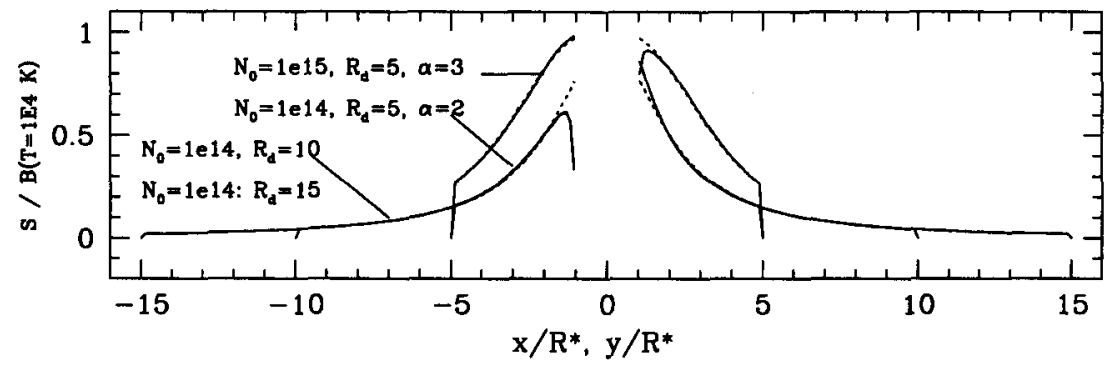

Figure 3. Source functions along the axis of highest amplitude in the equatorial plane of the disk for different model parameters.
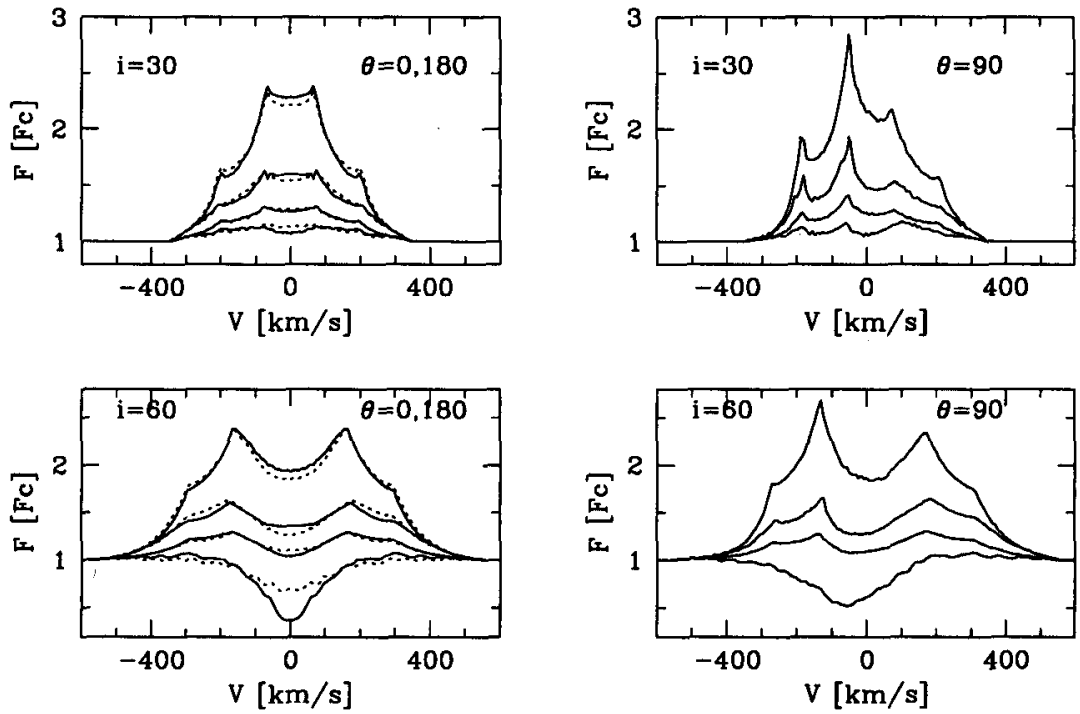

Figure 4. Theoretical emission line profiles for $\mathrm{H} \alpha$. In each panel we show model profiles of four models at a certain aspect angle defined by $(i, \theta)$. From top to bottom: $N_{0}=10^{15} \mathrm{~cm}^{-3}$ with $m=2, N_{0}=$ $10^{14} \mathrm{~cm}^{-3}$ with $m=2, N_{0}=10^{15} \mathrm{~cm}^{-3}$ with $m=3$, and $N_{0}=10^{13} \mathrm{~cm}^{-3}$ with $m=2$ but scaled by a factor of 5 for better visibility. The dotted profiles are at aspect angle of $\theta=180^{\circ}$. For $\theta=0^{\circ}$ the high density region is in front of the star. 

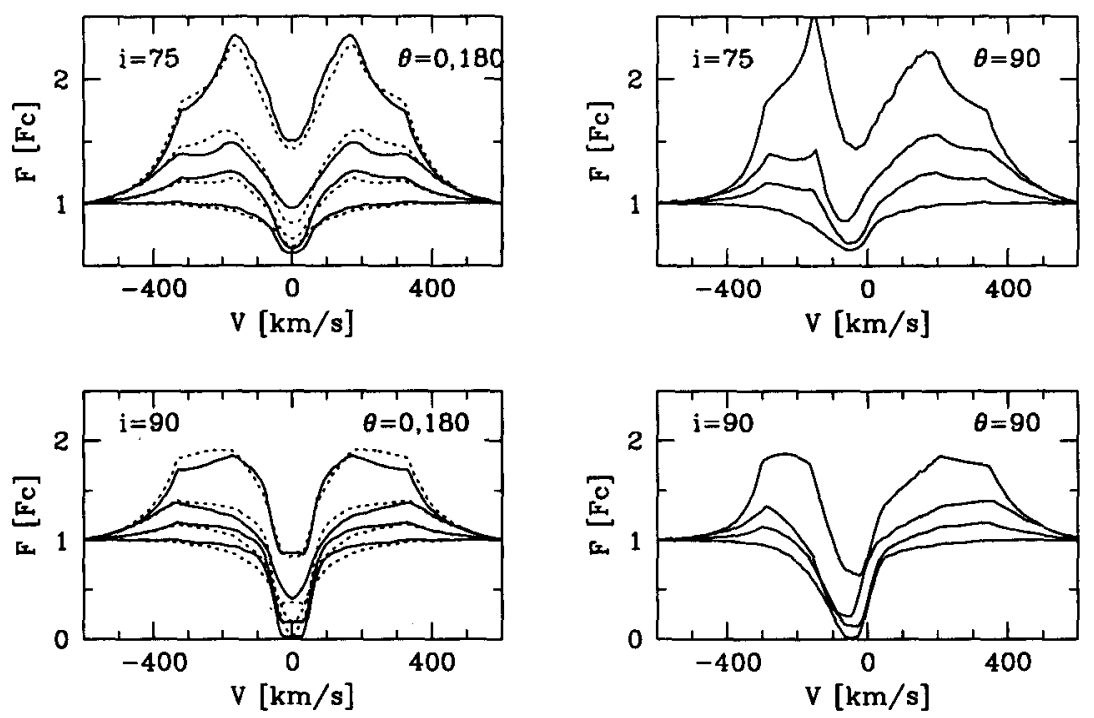

Figure 5. As Fig. 4, but for larger inclinations.

The resulting line profiles verify the suggestion that the combined interaction between kinematical broadening and broadening due to non-coherent scattering plays the same important role in class 2 profiles as for the class 1 profiles. The line profile fits (Fig. 6) show that both the shapes of asymmetric $\mathrm{H} \alpha$ line profiles as well as the cyclic time variability pattern can be reproduced by a model disk with a disturbed density structure as devised by Okazaki (1991).

As a systematic difference, model profiles are too faint with respect to observed emission lines and the bumps in the wings caused by the slab effect are stronger than observed. These discrepancies are caused by the underestimated disk radius of only $5 R_{*}$.

\subsection{Large disks}

In the meantime it has been found that the inclusion of the quadrapole moment in the external gravitational potential allows wave patterns become naturally confined within a few stellar radii (Papaloizou et al. 1992; Okazaki 1996). These new modifications do not require any longer the disk radius to confine the waves but the outer disk radius becomes again a free parameter. Furthermore, prograde motion of the density wave is permitted. Observational evidence for prograde pattern precession has been found by Telting et al. (1994) and Mennickent et al. (1997).

In order to account for these new theoretical results, and since the impact of the potential's quadrapole moment to the eigenmodes is rather small (Okazaki, 1996), we retain the wave structure for the disk within $5 R_{*}$ but append an unperturbed Keplerian disk at 5-10 and 5-15 stellar radii. With this constraints we 


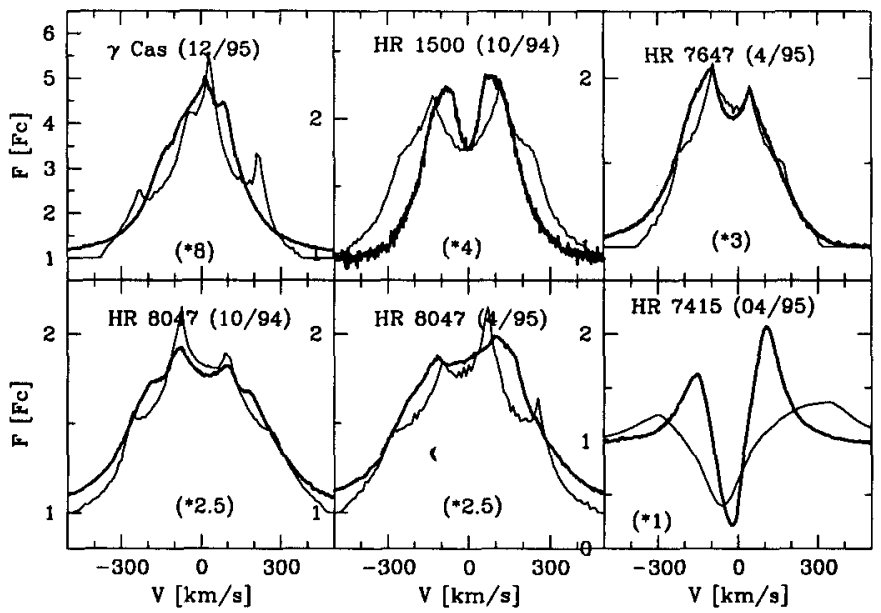

Figure 6. Comparison between observation and model profiles for small disks. Model profiles are scaled in intensity (see factor) to match observed line strengths.

consider Keplerian disks with a large $\mathrm{H} \alpha$ emitting area, where the superimposed density wave is confined to a few stellar radii as predicted. For the calculations we have tripled the number of gridpoints along both axes in the disk plane in the model with $R_{\mathrm{d}}=15$ in order to retain the numerical grid resolution used for the $R_{\mathrm{d}}=5$ model disks described above. The resulting converged line-source functions of different model disks are shown in Fig. 3. A set of $\mathrm{H} \alpha$ line profiles for a B5V star with $N_{0}=10^{14} \mathrm{~cm}^{-3}$ and disk radii $R_{\mathrm{d}}=5,10,15$ are given in Fig. 7.

The profiles from our second attempt (Fig. 8) now match the observed equivalent widths much better. A scale parameter to match observed line strengths is no longer required. Model profiles are also smoother with respect to the first attempt and agree better with the observed profile types. However, model profiles are on the whole less asymmetric than the observations, in particular at low inclinations. Hence the amplitude or the extent of the underlying perturbation is still too small. Since the amplitude factor in use is already large, we believe that a turnover radius of $5 R_{*}$ is too small to account for the observed amplitude. This suggestion supports the recent theoretical results by Okazaki (1996) and Savonije (1998) who both conclude that turnover radii of 10-20 $R_{*}$ can be expected. This means that the whole part of the disk visible in $\mathrm{H} \alpha$ can contributed to the line profile asymmetry.

\section{Conclusions}

We have shown that a vertically extended Keplerian disk can explain the observed class $1 \mathrm{H} \alpha$ emission lines of Be stars for the full range of stellar inclina- 


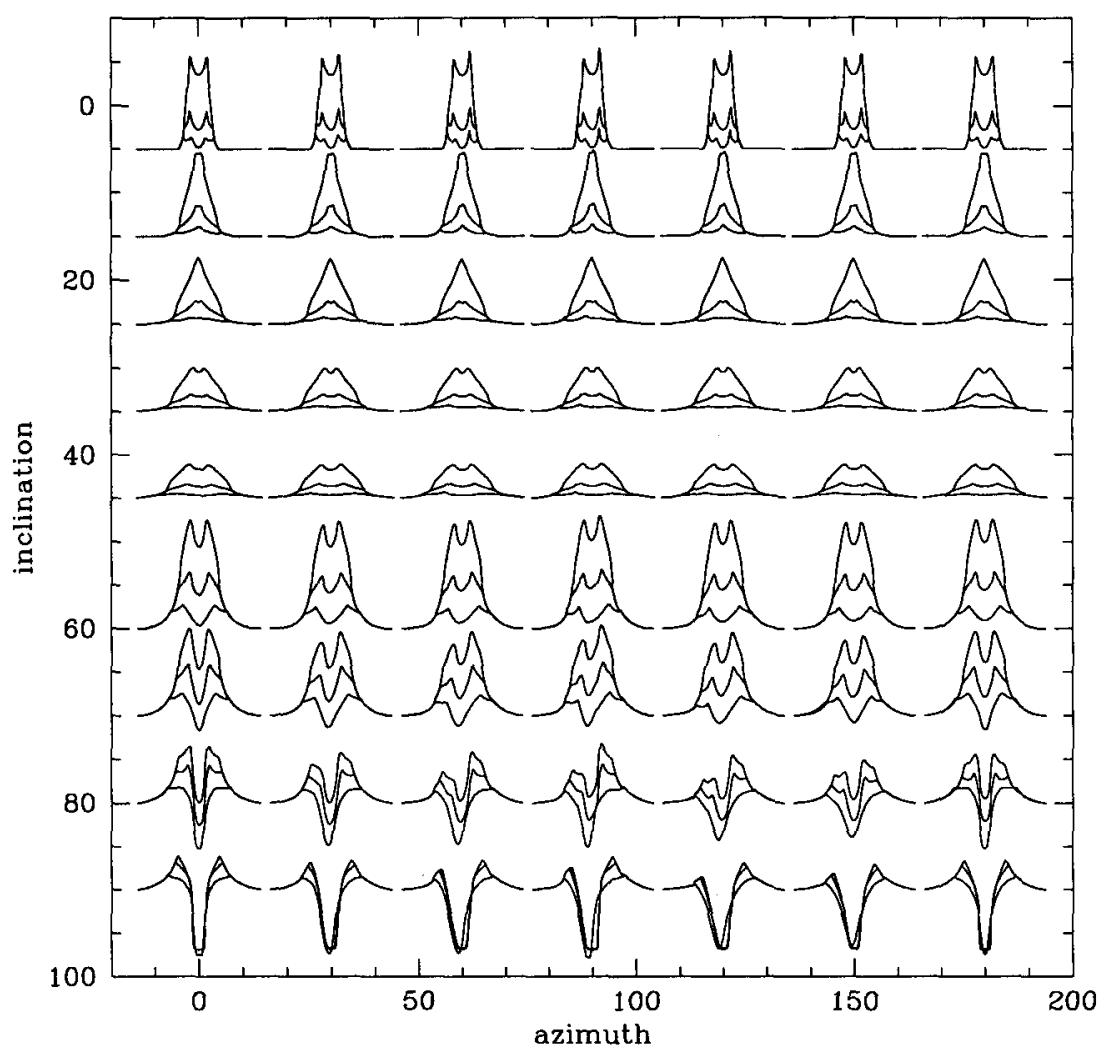

Figure 7. Theoretical $\mathrm{H} \alpha$ emission lines of non-axisymmetric Be star circumstellar disks. The profiles are ordered according the viewing angles $i$ inclination and $\phi$ (azimuth). E.g. The third set of three profiles in the first row is seen at $i=5^{\circ}$ and $\phi=60^{\circ}$. The strongest of the three profiles is for a disk extended up to $R_{\mathrm{d}}=15 R_{*}$, the lower one for $R_{\mathrm{d}}=10 R_{*}$, and the faintest for $R_{\mathrm{d}}=5 R_{*}$. All profiles are for a $M K=B 5 V$ central star. Two different scales have been adopted. For $i<50^{\circ}$ we used $F / F_{c}=\frac{1}{2}\left(\Delta i /^{\circ}\right) ; \Delta \lambda / \AA=\frac{1}{1.8}\left(\Delta \phi /^{\circ}\right)$. These low inclination profiles are plotted within the range of $\Delta \lambda= \pm 8 \AA$. For $i>50^{\circ}$ we used $F / F_{c}=\frac{1}{9}\left(\Delta i /^{\circ}\right) ; \Delta \lambda / \AA=\left(\Delta \phi /^{\circ}\right)$. The high inclination profiles are plotted within the range of $\Delta \lambda= \pm 14 \AA$. 


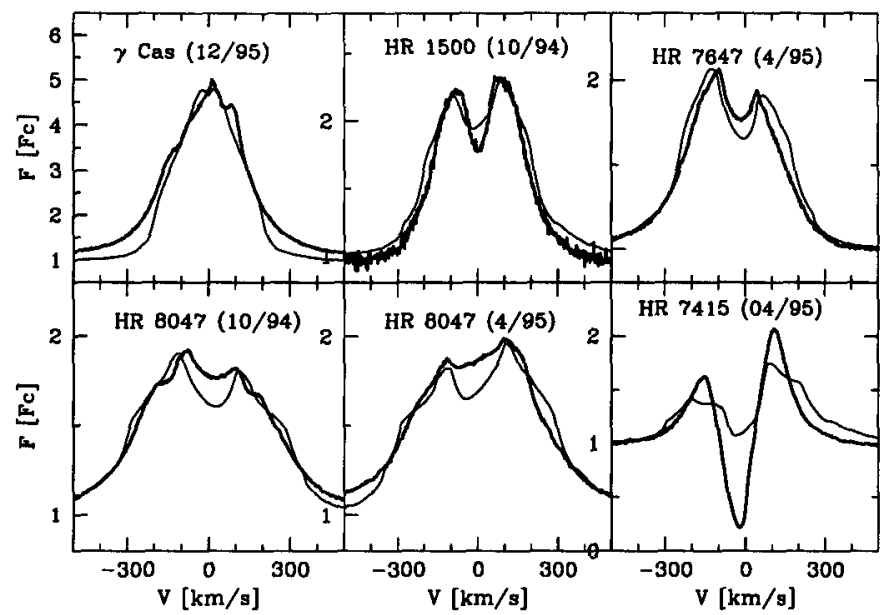

Figure 8. Comparison between observation and model profiles for large disks. A scale factor is no longer required to match observed line strengths.

tions. Additional outflow in the disk is not required, and in fact even contradicts the observations.

Using the one-armed global disk oscillations as an underlying structure for the radiative transfer we have shown that this scenario is capable of explaining the long-term variations of class 2 profiles. The exact extension of the perturbation in the disk is not yet fully known. However, comparison between observations and theory demonstrates that the perturbation is certainly spread over more than five stellar radii.

Acknowledgments. We thank the reader for his patience.

\section{References}

Ballereau, D., Chauville, J., 1989, A\&A, 214, 285

Doazan, V., Franco, M., Ruscini et al., 1987, ESA-SP-1147

Hanuschik, R. W., 1988, A\&A, 190, 187

Hanuschik, R. W., Hummel, W., Sutorius, E. et al., 1996, A\&AS, 116, 309

Hummel, W., 1994, A\&A, 289458

Hummel, W., Dachs, J., 1992, A\&A, 262, L17

Hummel, W., Hanuschik, R. W., 1997, A\&A, 320, 852

Horne, K., Marsh, T., 1986, MNRAS, 218, 761

Huang, S. S., 1972, ApJ, 171, 549

Huang, S. S., 1973, ApJ, 183, 541

Kato, S., 1983, PASJ, 35, 249 
Kriz, S., 1978, Bull. Astron. Soc. Czech., 30, 95

Mennickent, R. E., Sterken, C., Vogt, N., 1997, A\&A, 326, 1167

Okazaki, T., 1991, PASJ, 43, 75

Okazaki, T., 1997, A\&A, 318, 548

Papaloizou, J. C., Savonije, G. J., Henrichs, H. F., 1992, A\&A, 265, L45

Poeckert, R., 1982, in IAU Symp., 98, p453

Poeckert, R., Marlborough, J. M., 1978, ApJ, 220, 940

Quirrenbach, A., Bjorkman, K. S., Bjorkman, J. E. et al., ApJ, 479, 477

Rybicki, G. B., 1991, in NATO ASI Seies C, 341, p1

Savonije, G., 1998, in ESO Astrophys. Symp., XXII, p337

Smak, J., 1981, Acta Astron., 31, 395

Struve, O., 1931, ApJ, 73, 94

Telting, J. H., Heemskerk, M. H. M., Henrichs, H. F. et al., 1994, A\&A, 288, 558

\section{Discussion}

P. Harmanec: Intuitively I would expect that the choice of the boundary conditions for your 3D modeling may not be an easy task. At the same time I would expect that the choice of boundary conditions may strongly affect your result.

W. Hummel: The impact of geometrical and kinematical boundary conditions, such as the radius and the velocity field, on the line profiles is well known. The most critical boundary condition in the modeling is the excitation condition at the inner boundary of the $\mathrm{H} \alpha$-emitting disk.

P. Harmanec: Many investigators who tried to calculate Balmer emission line profiles since the 1960s have pointed out that it is relatively easy to model one Balmer line but that a critical test of the model is to compare the results with at least three different Balmer lines. How does your model perform in this respect? W. Hummel: Modeling many lines simultaneously requires the solution of the equations of statistical equilibrium for a multi-level model atom, which would increase computing time by several magnitudes.

P. Harmanec: Previous modeling showed that for stars with double peaked emission, the peak separation measures the outer radius of the disk quite well. You assume that the disks are Keplerian rotating. Applied to the disk in the binary star $\phi$ Per this leads to the result that the envelope is larger than the corresponding Roche lobe around the binary, which is hard to believe.

W. Hummel: The peak separation method implies Keplerian rotation which is valid for single Be stars. The circumprimary disk in the binary $\phi$ Per is not Keplerian but rather follows $V_{\text {rot }} \sim R^{-0.8}$ because of the Roche lobe geometry. Therefore the peak separation method would overestimize the outer disk radius. Using $V_{\text {rot }} \sim R^{-0.8}$ the resulting disk radius in $\phi$ Per is about $R_{\mathrm{d}}=10 R_{*}$, much smaller than $L_{1}$ at $19 R_{*}$.

Ph. Stee: I think you cannot ignore the expansion in the disk: rotation decreases as a function of the distance from the star whereas the expansion in- 
creases. So after a few stellar radii you are dominated by the stellar wind, where the $\mathrm{H} \alpha$ emission is also formed.

W. Hummel The observations clearly demonstrate that emission lines originate in a rotating disk without expansion. The outflowing and rotating wind does not contribute to the emission lines in the optical.

$\mathbf{P h}$. Stee I do not agree that electron scattering is capable of broadening the wings of the $\mathrm{H} \alpha$ line up to $1200 \mathrm{kms}^{-1}$. It is due to kinematical effects (mostly the wing effect).

W. Hummel: The broad wings of Balmer lines have been successfully modeled by electron scattering, see Poeckert \& Marlborough (1979).

Ph. Stee: From the computation of the one-armed oscillations line profiles it seems to me that the shape of the line is well reproduced but not the intensity (even if you need very high density in the photosphere, such as $10^{15} \mathrm{~cm}^{-3}$ ). Could you comment on that point?

W. Hummel: This is true for our first approach where we tried to fit observations with a $R_{\mathrm{d}}=5 R_{*}$ model disk. There we had to use large densities to compensate for the small radius. Line intensities from our recent model set with $R_{\mathrm{d}}=15 R_{*}$ agree well with observations.

J. Zorec: From your disk model, which has a uniform temperature, you have concluded that emission spectral lines have two peaks in two cases: (a) flat disks, (b) under given kinematical conditions. Evidence is piling up which shows that there is a strong activity in the stellar surface and in the transition region from the star to the circumstellar envelope. Assuming that this activity can be translated into some kind of chromospheric-corona like temperature structure, the consequent enhancement of the line source function also lead to emission spectral lines with two peaks, even the region of formation of lines is assumed to be spherical. If the region is expanding, the line profiles become non-symmetrical. Some line profiles produced by such a model are shown in the poster by Cidale et al., Paschen and Brackett lines in Be stars

W. Hummel: I will have a look at that poster.

J. Kubat: Why do you use Cartesian coordinates for the radiative transfer and not the cylindrical ones that seem to be more normal for the case of disks W. Hummel: For an axisymmetric model, cylinder coordinates are certainly advantageous to make use of the symmetries, e.g. by reducing the number of spatial dimensions. For non-axisymmetric disks, Cartesian coordinates become more advantageous.

L. Iliev: What would be the influence of a weak magnetic field expected in $\mathrm{Be}$ stars on the modeled line profiles.

W. Hummel: It would impact the velocity field in the region close to the star. Hence the high velocity part of the profile, the wings, would be influenced the most.

S. Owocki: You mentioned a difficulty in producing $V / R$ asymmetry at oblique median angles. Perhaps allowing the global disk oscillation to also affect the source function (e.g. through temperature) might help further.

W. Hummel: I agree. Maybe it is not necessarily the temperature, but simply an opacity effect. This ambiguity can only be resolved in a multi-level model atom. 